Facilitating the Implementation of EPC Reports in Learning Health Systems Engaged in Quality Initiatives: an EPC Pilot Project on ADHD 


\section{Facilitating the Implementation of EPC Reports in Learning Health Systems Engaged in Quality Initiatives: an EPC Pilot Project on ADHD}

Prepared for:

Agency for Healthcare Research and Quality

U.S. Department of Health and Human Services

5600 Fishers Lane

Rockville, MD 20857

www.ahrq.gov

Contract No. 290-2015-00013-I; 290-2015-00008-I

Prepared by:

Mayo Clinic Evidence-based Practice Center

Rochester, MN

Minnesota Evidence-based Practice Center

Minneapolis, MN

Investigators:

Allison S. Morrow, B.A.

Mary Butler, Ph.D., M.B.A.

M. Hassan Murad, M.D., M.P.H.

AHRQ Publication No. 19(20)-EHC029-EF

October 2019 


\section{Key Messages}

\section{Purpose of Report}

To evaluate and enhance the utility of an Evidence-based Practice Center (EPC) report by a health system engaged in QI (quality improvement).

\section{Key Messages}

- We developed an implementation guide based on a published EPC report to support Learning Health Systems engaged in QI initiatives.

- Post publication processing of EPC reports is needed to cater to a health system QI needs. It is difficult for EPC report authors to know in advance which information is most useful for a particular QI effort.

- A product with narrow focus is critical for QI initiatives. EPC reports are large and cover a variety of interventions and outcomes. It is overwhelming to stakeholders to find and apply the information in QI initiatives.

- Engagement of stakeholders is essential to determine what information is important to health systems at a given time.

- Data need to be re-extracted from the studies included in the EPC report.

- Additional sources of data from outside the report are commonly required. Cost information is a clear example. 
This report is based on research conducted by the Mayo Clinic and Minnesota Evidence-based Practice Center (EPC) under contract to the Agency for Healthcare Research and Quality (AHRQ), Rockville, MD (Contract No.290-2015-00013-I;290-2015-00008-I ). The findings and conclusions in this document are those of the authors, who are responsible for its contents; the findings and conclusions do not necessarily represent the views of AHRQ. Therefore, no statement in this report should be construed as an official position of AHRQ or of the U.S. Department of Health and Human Services.

\section{None of the investigators have any affiliations or financial involvement that conflicts with the material presented in this report.}

The information in this report is intended to help healthcare decision makers-patients and clinicians, health system leaders, and policymakers, among others-make well-informed decisions and thereby improve the quality of healthcare services. This report is not intended to be a substitute for the application of clinical judgment. Anyone who makes decisions concerning the provision of clinical care should consider this report in the same way as any medical reference and in conjunction with all other pertinent information, i.e., in the context of available resources and circumstances presented by individual patients.

This report is made available to the public under the terms of a licensing agreement between the author and the Agency for Healthcare Research and Quality. This report may be used and reprinted without permission except those copyrighted materials that are clearly noted in the report. Further reproduction of those copyrighted materials is prohibited without the express permission of copyright holders.

AHRQ or U.S. Department of Health and Human Services endorsement of any derivative products that may be developed from this report, such as clinical practice guidelines, other quality enhancement tools, or reimbursement or coverage policies, may not be stated or implied.

Persons using assistive technology may not be able to fully access information in this report. For assistance contact EPC@ahrq.hhs.gov.

Suggested citation: Morrow AS, Butler M, Murad MH. Facilitating the Implementation of EPC Reports in Learning Health Systems Engaged in Quality Initiatives: an EPC Pilot Project on ADHD. Methods Research Report. (Prepared by the Mayo Clinic and Minnesota Evidence-based Practice Center under Contract No. 290-2015-00013-I; 290-2015-00008-I.) AHRQ Publication No. 19(20)-EHC029-EF. Rockville, MD: Agency for Healthcare Research and Quality; October 2019. Posted final reports are located on the Effective Health Care Program search page. DOI: http://doi.org/10.23970/AHRQEPCMETHQUALIMPRFACILITATING. 


\section{Preface}

The Agency for Healthcare Research and Quality (AHRQ), through its Evidence-based Practice Centers (EPCs), sponsors the development of evidence reports and technology assessments to assist public- and private-sector organizations in their efforts to improve the quality of healthcare in the United States. The reports and assessments provide organizations with comprehensive, science-based information on common, costly medical conditions and new healthcare technologies and strategies. The EPCs systematically review the relevant scientific literature on topics assigned to them by AHRQ and conduct additional analyses when appropriate prior to developing their reports and assessments.

To improve the scientific rigor of these evidence reports, AHRQ supports empiric research by the EPCs to help understand or improve complex methodologic issues in systematic reviews. These methods research projects are intended to contribute to the research base in and be used to improve the science of systematic reviews. They are not intended to be guidance to the EPC program, although may be considered by EPCs along with other scientific research when determining EPC program methods guidance.

AHRQ expects that the EPC evidence reports and technology assessments will inform individual health plans, providers, and purchasers as well as the healthcare system as a whole by providing important information to help improve healthcare quality. The reports undergo peer review prior to their release as a final report.

If you have comments on this Methods Research Project they may be sent by mail to the Task Order Officer named below at: Agency for Healthcare Research and Quality, 5600 Fishers Lane, Rockville, MD 20857, or by email to epc@ahrq.hhs.gov.

Gopal Khanna, M.B.A.

Director

Agency for Healthcare Research and Quality

Stephanie Chang, M.D., M.P.H.

Director

Evidence-based Practice Center Program

Center for Evidence and Practice Improvement

Agency for Healthcare Research and Quality
Arlene Bierman, M.D., M.S.

Director

Center for Evidence and Practice Improvement

Agency for Healthcare Research and Quality

Jill Huppert, M.D.

Task Order Officer

Center for Evidence and Practice Improvement

Agency for Healthcare Research and Quality 


\section{Acknowledgments}

The authors gratefully acknowledge the following individuals for their contributions to this

project: Joan Bissen, R.D., Rachel Lynch, M.D., Kevin Shaw, Leslie Sim, Ph.D., Andrea Singh, M.D., and Stephan Whiteside, Ph.D. 


\section{Facilitating the Implementation of EPC Reports in Learning Health Systems Engaged in Quality Initiatives: an EPC Pilot Project on ADHD}

\section{Structured Abstract}

Background. Evidence synthesis reports prepared by the Evidence-based Practice (EPC) program are critical to providing evidence for clinical practice and guideline development. However, from the perspective of a health system focused on quality improvement (QI), such large evidence reports with wide scope are difficult to implement within a QI initiative.

Methods. We consulted with two health systems to identify a published EPC report on a topic of interest to future quality initiatives. After identifying the target report on attention deficit hyperactivity disorder (ADHD), we gathered feedback from the health system to determine what information on ADHD treatments is the most relevant to their quality initiatives and the most useful way to deliver this information. We then developed a prototype product derived from the original EPC report, supplemented by newly abstracted contextual material from the included studies as well as outside resources. We presented the tool to representatives from two health systems and two parents of children with ADHD to obtain feedback and modify/enhance the product in an iterative fashion. A designer assisted in improving the usability, readability, and presentation of the product.

Results. Stakeholders requested practical information to support applying the findings of the evidence report, including a parent and family education guide, resources to recommend for parents, current best practices from leading practitioners, and advise on medication management. We developed a parent and family education guide that included example curricula based on three randomized trials identified by the report. Cost information was explicitly requested but was difficult to obtain. Health system stakeholders and parents of children valued the guide, suggesting possible new products for the EPC program. The lessons learned from this pilot project include; (1) Post publication processing of EPC reports is needed to cater to a health system QI needs, (2) A product with narrow focus is critical for QI initiatives, (3) Engagement of stakeholders is essential, (4) Data need to be re-extracted from included studies, and (5) Additional sources of data from outside the report are commonly required.

Conclusion. An implementation tool derived from an EPC report and developed with stakeholder feedback may facilitate implementation of evidence in a health system engaged in a quality improvement initiative. 


\section{Contents}

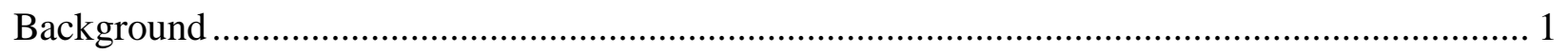

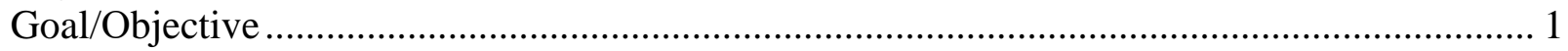

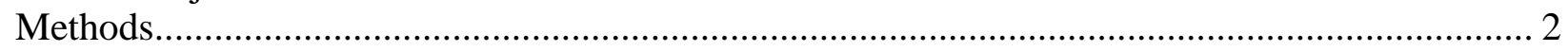

Health System Description ................................................................................................ 2

Topic Identification ........................................................................................................... 2

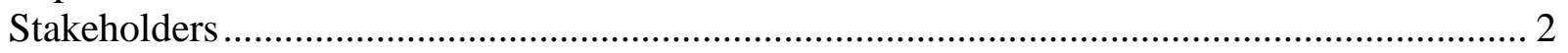

Development of the Tool and Subsequent Modification and Evaluation ..................................... 2

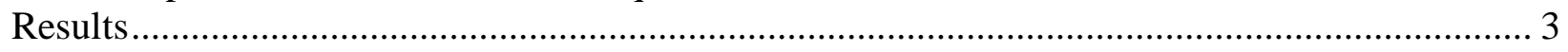

Initial Feedback and Selection of Focus …………............................................................... 3

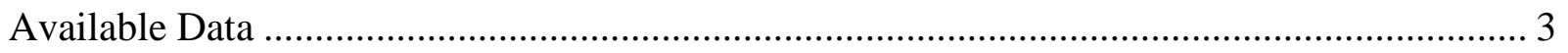

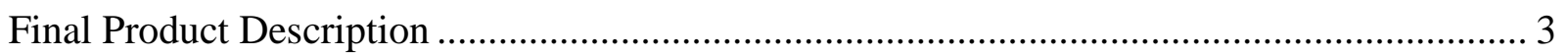

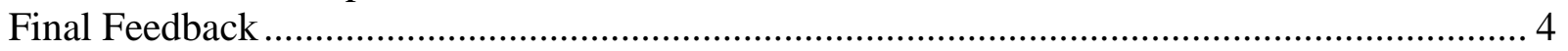

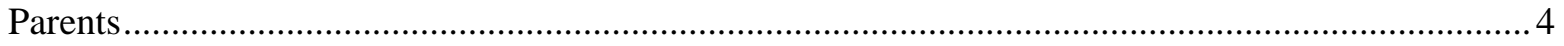

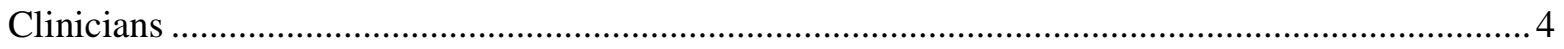

Health System Stakeholders...................................................................................................... 4

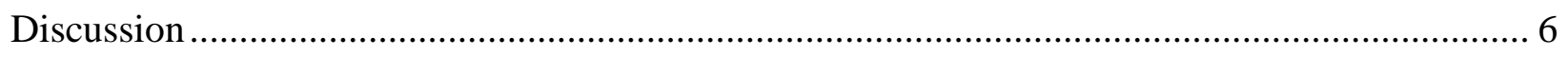

Lessons Learned and Applicability for other EPC Reports ......................................................... 6

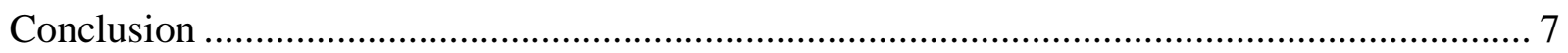

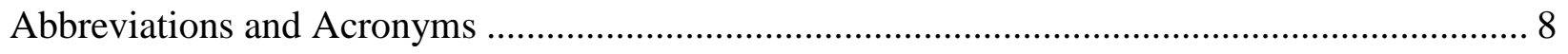

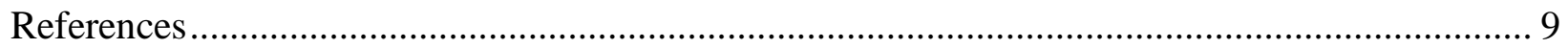

\section{Figures}

Figure 1. Steps followed to facilitate using EPC reports in LHS QI initiatives ............................. 7

\section{Appendixes}

Appendix A. Implementation Guide 


\section{Background}

Evidence synthesis reports prepared by the Evidence-based Practice (EPC) program are critical to providing evidence for clinical practice and guideline development. However, from the perspective of a health system focused on quality improvement, such large evidence reports with wide scope are difficult to implement. Improving report implementation requires tailoring the presentation, selecting a focused topic of current relevance to the health system initiatives, and creating implementation tools that provide contextual and practical information.

Relevant implementation and contextual information may be present in original EPC reports and attached appendices. However, other available information may be useful for implementation purposes. Unabstracted relevant information may be present in the included study publications. Additional contextual information not necessary for the original EPC report may also be obtained from other sources (published literature or unpublished documents and webpages).

This exposition describes a combined effort of two EPCs, Mayo Clinic and Minnesota, in which an existing EPC report was used to develop an evidence synthesis product (an implementation guide or a tool) to assist health systems interested in a quality improvement (QI) project in the clinical area of the EPC report. This project was initially motivated by an interest in whether an implementation tool could be developed that would be generally useful to health systems and interested stakeholders. Another important question is whether such tools are valued by more than a single health system.

\section{Goal/Objective}

The primary goal of this project is to create an evidence product for health systems to help them utilize the results of an EPC report. To reach that goal we have the following objectives:

- To identify what information in an EPC report is important for a health system engaged in a quality improvement initiative.

- To develop an evidence product (i.e., tool) that delivers information in a practical way to a health system.

- To iteratively enhance/refine the tool based on feedback from health systems. 


\section{Methods}

\section{Health System Description}

We engaged two healthcare systems, HealthPartners and Mayo Clinic. Founded in 1957 as a cooperative, HealthPartners is an integrated healthcare organization providing healthcare services and health plan financing and administration. HealthPartners has 8 hospitals and more than 90 clinics offering 55 specialties. HealthPartners is affiliated with the University of Minnesota Learning Health System Network for an Agency for Healthcare Research and Quality K12 training grant, but is otherwise a community-based, not academic, health system. The Mayo Clinic Health System is a family of clinics, hospitals and other healthcare facilities serving more than 60 communities in Minnesota, Wisconsin and Iowa. Founded in 1992, the system links the expertise of Mayo Clinic with healthcare providers in local communities to offer patients a full spectrum of healthcare options, with more than 100 medical services and specialties available throughout the system.

\section{Topic Identification}

We aimed to identify ongoing or planned QI effort in the clinical areas of recently published EPC reports (2015-present). After compiling a list of eligible EPC reports, we queried QI specialists from both health systems to see if any eligible topic matched a QI endeavor. The Mayo Clinic had a registry of QI projects from which we screened over 2,000 projects. We found no match between eligible topics and on-going QI efforts. However, HealthPartners expressed interest in the topic of attention deficit-hyperactivity disorder (ADHD) as a potential future focus. Therefore, the ADHD topic was chosen for this pilot. ${ }^{1}$

\section{Stakeholders}

Feedback was obtained from (1) a health system executive leading QI efforts at HealthPartners, (2) two parents with children who have ADHD (both recruited from Mayo Clinic), (3) two pediatricians with expertise/career focus on ADHD (one from each health system), (4) two mental health providers (Mayo Clinic) and (5) a designer with expertise in developing communication tools and educational materials for patients and the public (Mayo Clinic).

\section{Development of the Tool and Subsequent Modification and Evaluation}

We used feedback from stakeholders to narrow the focus from that of the report to a specific area relevant to future QI effort. We searched the EPC report for contextual and practical implementation information about the chosen focus. We extracted data from the original trials included in the report and developed a product in print.

A draft of this product was presented to stakeholders for feedback which led to modifications and enhancement in an iterative process. The product was also evaluated by a designer who suggested changes for usability and readability. Additionally, two parents provided feedback. 


\section{Results}

\section{Initial Feedback and Selection of Focus}

The health system stakeholders was asked about the EPC report ${ }^{1}$ and what suggestions they had to make it more useful. They were concerned about the size of the report and the breadth of interventions and key questions, describing it as "overwhelming and impenetrable." They thought that most of the information was already known and did not increase their current knowledge of ADHD. They suggested directing separate key messages to researchers and clinicians. The stakeholders requested practical information to support applying the results. These included resources to recommend for parents, current best practices from leading practitioners, and medication management (dosing, adverse events, and medication vacations).

Parent education programs were highlighted by the health system stakeholder multiple times. They voiced a great need for practical, evidence based information about ADHD for parents and families that would obviate the need for searching the Internet and encountering sources that were untrustworthy. They expressed the need for information about strategies for parenting children with ADHD. Parents wanted information to assist in their understanding of the diagnosis, prognosis, symptoms, and treatment of ADHD, as well as guidance on treatment options, handling adverse events from medication, and managing the child's behaviors.

\section{Available Data}

The EPC report demonstrated that parent training and education programs improved ADHD symptoms in children aged 7 to 17 years old. An earlier 2011 report on ADHD found this was also true for children younger than 6 years old. ${ }^{2}$ The EPC report focused on comparative effectiveness and did not provide sufficient description of these programs. We evaluated other behavioral interventions covered in the report that might provide further contextual information regarding training and education programs, even though the interventions themselves were assessed to have insufficient evidence. However, these interventions were, unsurprisingly, too briefly reported to be useful.

From six trials of effectiveness of parent and education program, we chose 3 trials that had low risk of bias, provided adequate description of their curricula, and showed the curricula to be effective in reducing ADHD symptoms, improving parents' knowledge and improving

medication adherence. ${ }^{3-5}$ We extracted details of the interventions (population included, frequency and duration of intervention, who provided the intervention, how families were approached, and the content and format of the intervention).

\section{Final Product Description}

The final product was a guide on developing a curriculum for parents and families of children with ADHD.

This guide was presented as a hard copy, brochure style two page document. It was produced using Microsoft Publisher (see Appendix). There are 7 headings (Rational and Effectiveness, Who is eligible, How to approach families, How long and how often, Provided by, Helping parents learn, Examples of curriculum format). Additionally an example of a 12 week curriculum from one of the original trials was included. The back page lists the references used to create this guide as well as Agency for Healthcare Research and Quality disclaimers. The main content of the guide is approximately 500 words without the disclaimers and references. 


\section{Final Feedback}

\section{Parents}

The guide was met with great enthusiasm by parents of children with ADHD. One parent stated that the information provided in this parent training program is "desperately needed." Parents mentioned they tried to do their own research online and found it overwhelming. They greatly desired an education program and expressed interest in participating in a future QI initiative in this area. Another parent suggested including the children in some of the sessions, allowing them to be more involved in their treatment and not rely on their parents to relay information to them. They also suggested sessions include siblings to increase family awareness of ADHD.

\section{Clinicians}

A primary care physician who specializes in ADHD from the Mayo health system was interviewed about this implementation guide. She found the handout very helpful and mentioned the importance of training and education for parents of children with ADHD. She was concerned about the applicability of this research to the real world. For example, the parent training curriculum in the trials was provided by psychologists and psychiatrists whereas in practice, it is often primary care physicians that regularly treat ADHD. Education in community practice is often provided by clinicians, nurses and social workers. The clinician also mentioned that similar education trainings at Mayo Clinic are provided in blocks of four weeks so that parents are not committed to 12 consecutive weeks of the program. The clinician cited possible challenges to implementation such as cost, reimbursement, space, and personnel, and suggested online delivery formats as being more feasible. Overall, the handout was very well received by the clinician. She said that it was helpful in guiding physicians to choose the topics in which parents are seeking information.

A psychologist also reviewed the implementation guide. She suggested adding more details to the curriculum that would aid primary care physicians in better understanding the information that should be presented to parents. These additions would include specific parenting techniques (ex. praise, ignoring, breaks, rewards) as well as what nonpharmacological treatments are evidence-based. It was also recommended to move sessions on pharmacologic and nonpharmacologic treatment to the end of the curriculum. She thought it would be beneficial to include children in parent technique classes for more real-world instruction. The psychologist also recommended books and other resources on parent training. She liked the look of the guide and thought it would be a helpful tool.

\section{Health System Stakeholders}

The stakeholders overall liked the guide, found it helpful and focused, and were interested in implementing it as a part of a QI initiative. They requested additional information on the cost and reimbursement potential for such parent and family intervention. Since the included trials were not based in the U.S., we did not seek cost or cost-effectiveness data from these trials. We also did not contact trial authors for the same reason. Additional searches to find articles that addressed the cost of ADHD treatment were done and did not reveal costs associated with parent and family education programs. Cost information on similar programs in other conditions are available and demonstrate increasing cost per session if delivered by a nurse, a social worker, a 
Ph.D. psychologist, or a psychiatrist (which will most likely be the same for a general practitioner). 


\section{Discussion}

In this pilot project, we developed an implementation guide about ADHD parent and family education. This guide was intended to support a future QI initiative by a health system and may suggest a general approach that would be widely valuable to other health systems.

EPC reports are overwhelming to health system stakeholders and may be difficult to implement within a QI initiative. Health system stakeholders need the information tailored to their needs and delivered in short, succinct formats that focus on practical application of the data.

EPC reports focus on comparative effectiveness and cater to a wide audience. It is not feasible to extract every possible data in EPC reports because the exact stakeholder is not always known and their focus and specific information need is not always clear. Therefore, a process to create a tool or a product after the publication of the report will likely be needed most of the time to facilitate implementation, particularly to support a QI initiative. QI initiatives often start with a needs assessment and development of a charter; which is almost always unknown at the time of EPC report writing.

Cost and cost effectiveness remain a challenge to implementation. In this specific example, we knew that parent education programs improve ADHD symptoms and are desired by parents and clinicians, yet, from a health system perspective, it is critical to know the resources needed for implementation. We found the published literature about cost lacking. More importantly, health systems may find the societal cost used for these cost-effectiveness studies for individual children with ADHD less relevant. Since there is a higher likelihood that a health system is also providing care for the family, cost effectiveness may need to consider family system costs instead of individual child health costs. The economic burden of caring for a child with ADHD over the course of child development was estimated at 5 times the cost of youth without ADHD. In addition, parents were more likely to experience employment difficulties. ${ }^{6}$ Caregiver strain and resulting health impacts could be non-trivial. Unfortunately, these indirect costs remain unexamined.

\section{Lessons Learned and Applicability for Other EPC Reports}

- Post publication processing is needed: It is difficult for EPC report authors to know which information is most useful for a particular QI effort. Therefore, it seems that a process after the publication of the report is needed to develop a tool or a tailored presentation to facilitate use of evidence in QI initiatives.

- A product with narrow focus is critical for QI: EPC reports are large and cover a variety of interventions and outcomes. It is overwhelming to stakeholders to find and apply the information in QI initiatives. Thus, a tool (or a tailored presentation) with very narrow focus is needed for a particular QI initiative.

- Engagement of stakeholders is essential: It is important to determine what information is important to health systems at a given time to support a specific QI initiative. This is possible through engagement and interviews with relevant stakeholders throughout the tool development process.

- Data need to be re-extracted from included studies: The information needed for implementation is commonly not available in the EPC report. Re-extraction of data from the included relevant studies is needed to get more specific information. 
- Additional sources of data from outside the report are commonly required: Some information will not be available in the original studies summarized in the report. Outside sources are needed to get these data. Cost is a clear example.

The process used in this pilot is depicted in Figure 1.

Figure 1. Steps followed to facilitate using EPC reports in LHS QI initiatives

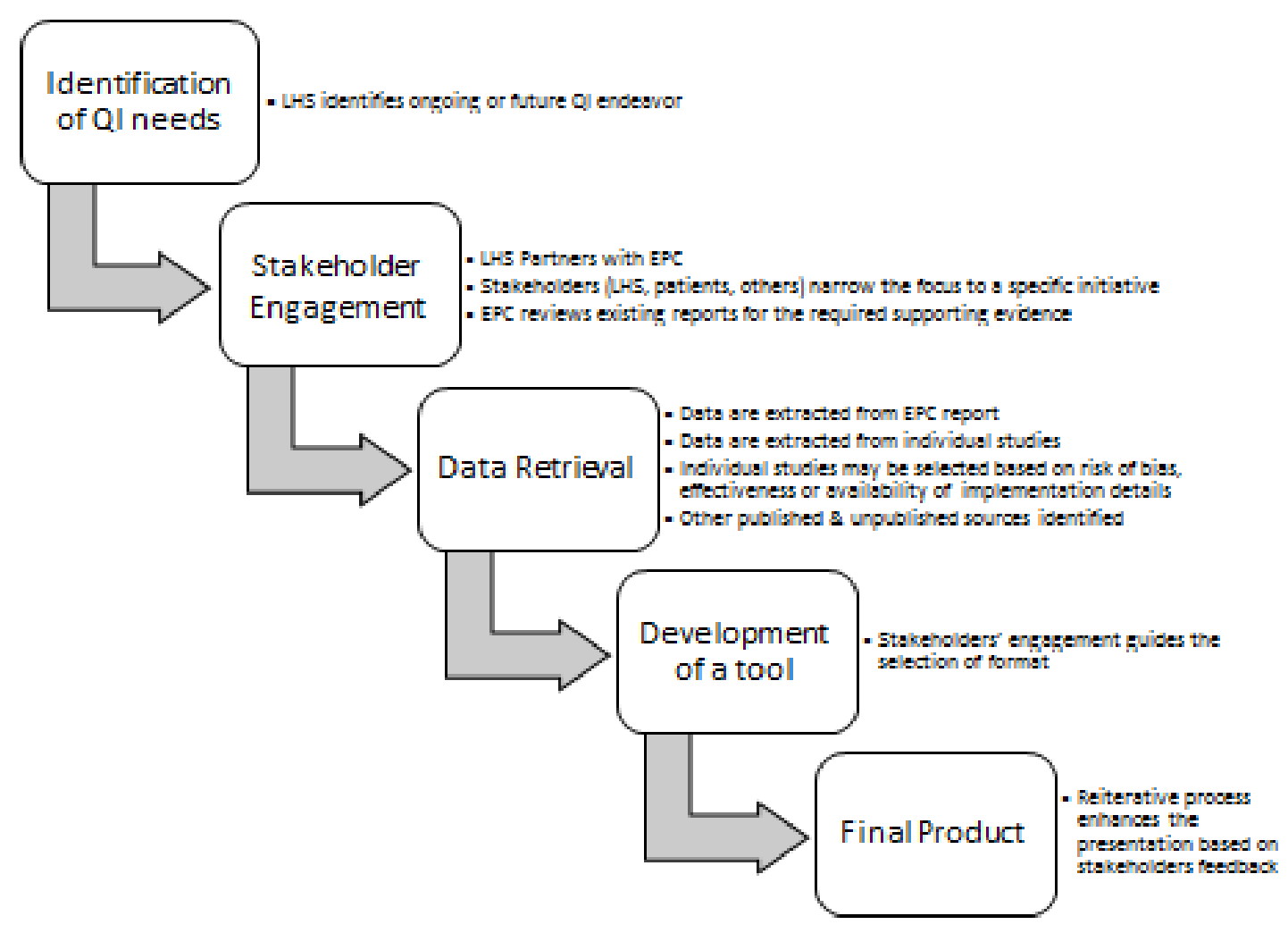

\section{Conclusion}

An implementation tool derived from an EPC report and developed with stakeholder feedback may facilitate implementation of evidence in a health system engaged in a quality improvement initiative. 


\section{Abbreviations and Acronyms}

ADHD

EPC

QI
Attention-Deficit/Hyperactivity Disorder

Evidence-based Practice Center

Quality Improvement 


\section{References}

1. Kemper AR, Maslow GR, Hill S, et al. AHRQ Comparative Effectiveness Reviews. Attention Deficit Hyperactivity Disorder: Diagnosis and Treatment in Children and Adolescents. Comparative Effectiveness Review No. 203. (Prepared by the Duke University Evidence-based Practice Center under Contract No. 290-201500004-I.) AHRQ Publication No. 18-EHC005-EF. Rockville, MD: Agency for Healthcare Research and Quality; January 2018. DOI: https://doi.org/10.23970/AHRQEPCCER203 .

2. Charach A, Dashti B, Carson P, et al. AHRQ Comparative Effectiveness Reviews. Attention Deficit Hyperactivity Disorder: Effectiveness of Treatment in At-Risk Preschoolers; Long-Term Effectiveness in All Ages; and Variability in Prevalence, Diagnosis, and Treatment. Comparative Effectiveness Review No. 44. (Prepared by the McMaster University Evidencebased Practice Center under Contract No. MME2202 290-02-0020.) AHRQ Publication No. 12-EHC003-EF. Rockville, MD: Agency for Healthcare Research and Quality. October 2011.. https://effectivehealthcare.ahrq.gov/products/adhd /research.
3. Bai G-n, Wang Y-f, Yang L, et al. Effectiveness of a focused, brief psychoeducation program for parents of ADHD children: improvement of medication adherence and symptoms. Neuropsychiatr Dis Trea. 2015;11:2721.

4. Ferrin M, Moreno-Granados J, Salcedo-Marin M, et al. Evaluation of a psychoeducation programme for parents of children and adolescents with ADHD: immediate and long-term effects using a blind randomized controlled trial. Eur Child Adolesc Psychiatry. 2014;23(8):637-47.

5. Ferrin M, Perez-Ayala V, El-Abd S, et al. A Randomized Controlled Trial Evaluating the Efficacy of a Psychoeducation Program for Families of Children and Adolescents With ADHD in the United Kingdom: Results After a 6Month Follow-Up. J Atten Disord. 2016 Feb 2. doi: 10.1177/1087054715626509. PMID: 26838557.

6. Zhao X, Page TF, Altszuler AR, et al. Family Burden of Raising a Child with ADHD. J Abnorm Child Psychol. 2019 August 01;47(8):1327-38. doi: 10.1007/s10802-019-00518-5. 


\section{Appendix A. Implementation Guide}

\section{IMPLEMENTATION GUIDE}

\section{Parent and Family Education for Children with ADHD}

\section{Rationale and effectiveness}

- Clinical practice guidelines recommend psychoeducation programs as a part of multimodal management for children with ADHD

- Parent and family educational programs reduces ADHD symptoms (total symptoms, inattention/cognition and hyperactivity/impulsivity subdomains) over 6 months follow up

- Parent and family educational programs improve medication adherence

Who is eligible...

Parents of children who are:

- Boys and girls age 3-19 years

- Have confirmed diagnosis of ADHD

- May be on medications (In studies, 60-100\% were on medications)

How to approach families...

- By phone as a part of a health system quality initiative

- In person when children presents to ADHD follow up appointments 


\section{How long and how often...}

- Option 1: Two- hour expert guided didactics provided weekly over 6 weeks

- Option 2:Two 40 minute group sessions, and online community engagement allowing parents to communicate with each other and with a professional counselor, over 3 months

\section{Provided by...}

- Health Educators (nurses and social workers)

- Primary Care Providers

- Psychologists

- Psychiatrists

\section{Helping parents learn...}

- Symptoms and medication adverse events

- Parenting skills and strategies to manage symptoms and reduce defiant behavior

- Barriers to treatment adherence and how to cope with them

- Best practices of parent and clinician relationship

- Results of relevant research

\section{Examples of curriculum format...}

- Manual for administering personnel

- Manual for parents after each session

- Brief homework for parents between sessions

- Forms for parents to track child's use of medication and adverse events 


\section{Example curriculum (Ferrin 2016)}

SEssion 1

Presentations and group functioning rules

What is ADHD? Definition of ADHD and gen eral overview of the different sessions

SESSION 2

Core symptoms: hyperactivity, inattention, and impulsivity

- What is ADHD and what is not?

- Diagnostic procedures. ADHD subtypes: inattentive, hyperactive/impulsive, and combined

SESSION 3

Etiological, maintaining, and perpetuating factors.

- Genes, environment as etiological factors.

- Myths, misconceptions, and stereotypes a bout ADHD

SESSION 4

Co-morbidities in ADHD.

Depressive and anxiety disorders, oppositional defiant disorder and conduct disorder, substance abuse, tic disorders, and learning disabilities

SESSION 5

Prognosis and outcome: ADHD in the adolescent and the adult.

Evolution of symptoms throughout the life span.

Executive function problems

\section{SESsion 6}

Non-pharmacological treatments: diets, supplements, cognitive, behavioral treatments, and neurofeedback.

What is really effective for ADHD? Scientific evidence of the different psychosocial

$$
\text { approaches }
$$

SESSION 7

Dealing with everyday-life problems at Home I.

Basic parenting techniques

SESSION 8

Dealing with everyday-life problems at Home II. Managing the child with difficult behaviors

SESSION 9

Dealing with everyday-life problems at school.

Helping the ADHD child at school/ with their homework

SESSION 10

Pharmacological treatments: stimulants fects Mode of action, monitoring, and side ef-

SEssion 11

Pharmacological treatments: non-stimulants. Mode of action, monitoring, and side effects

SESSION 12

Summarizing, final questions, and doubts. Closing down session. Feedback from parents 


\section{Additional resources:}

Barkley RA, Benton CM. Your defiant child: Eight steps to better behavior: Guilford Press; 2013.

- Webster Stratton C. The Incredible Years: a trouble shooting guide for parents of young children aged 3-8 years. Ontario: Umbrella Press; 2004.

- Eyberg S. Parent-child interaction therapy: Integration of traditional and behavioral

This implementation guide is based on a systematic review of the literature "Attention Deficit Hyperactivity Disorder: Diagnosis and Treatment in Children and Adolescents. Rockville (MD): Agency for Healthcare Research and Quality (US); 2018 Jan. "

This document is based on research conducted under contract to the Agency for Healthcare Research and Quality (AHRQ), Rockville, Maryland. The findings and conclusions in this document are those of the authors, who are responsible for its contents; the findings and conclusions do not necessarily represent the views of AHRQ. Therefore, no statement in this report should be construed as an official position of AHRQ or of the U.S. Department of Health and Human Services. None of the investigators have any affiliations or financial involvement that conflicts with the material presented in this report. The information in this document is intended to help health care decision makers-patients and clinicians, health system leaders, and policymakers, among others-make well-informed decisions and thereby improve the quality of health care services. This is not intended to be a substitute for the application of clinical judgment. Anyone who makes decisions concerning the provision of clinical care should consider this report in the same way as any medical reference and in conjunction with all other pertinent information, i.e., in the context of available resources and circumstances presented by individual patients. AHRQ or U.S. Department of Health and Human Services endorsement of any derivative products that may be developed from this document, such as clinical practice guidelines, other quality enhancement tools, or reimbursement or coverage policies, may not be stated or implied.

Data were derived from randomized trials judged to have the lowest risk of bias in the systematic review that provided detailed description of interventions that led to improvement in patient outcomes.

1. Bai GN, Wang YF, Yang L, et al. Effectiveness of a focused, brief psychoeducation program for parents of ADHD children: Improvement of medication adherence and symptoms. Neuropsychiatr. Dis Treat. 201 5;11:2721-35.

2. Fercin M, Perez-Ayala V, El-Abd S, et al. A Randomized Controlled Trial Evaluating the Efficacy of a Psychoeducation Program for Families of Children and Adolescents With ADHD in the United Kingdom: Results After a 6-Month Follow-Up. J Atten Disard. 2016 Feb 2doi: 10.1177/1087054715626509. PMID: 26838557.

3. Ferin M, Moreno-Granados JM, Salcedo-Marin MD, et al. Evaluation of a psychoeducation programme for parents of children and adolescents with ADHD: immediate and long-term effects using a blind randomized controlled trial. Fur Child edples, Psychiatry. 2014 Aug; $23(8): 637 \cdot 47$. dgi: 10.1007/300787-013-0494-7. PMID: 24292412. 